\title{
Perestroika and personality: Transformation of gender stereotypes amongst the youngsters in Russia
}

\author{
Vladimir Uryvaev \\ Demidov Yaroslavl State University, Russian Federation \\ Yaroslavl State Medical University, Russian Federation
}

\begin{abstract}
Introduction. The dynamics of gender stereotypes amongst the youngsters can provide important evidence about the tendencies in the changes of behavioural stereotypes in society. Goal. To conduct a psychodiagnostic evaluation of the changes in basic personal characteristics of young students (boys and girls) caused by the perestroika processes in the Russian society. Results. The comparison of two groups of medical students (both groups are identical: the second course, the department of general medicine, Yaroslavl State Medical University. Testing of 1992 and 2006 using the 16-PF Questionnaire and MMI - J. Nuttin) reveals at least two main tendencies in the aspect of gender differences. The first tendency is the advance increase of girls' adaptive capabilities compared to boys. The changes in girls involve substantially enhanced dominance and efficiency of self-regulation. The second notable tendency is a considerable review of values (J. Nuttin) in the "perestroika generation". The values of selfrealization, work, and social motivation are increased in both boys and girls. However, girls report substantially lower manifestation of self-analysis and value of passive recreation, while boys show the decreased value of cognitive and research motivation, as well as "transcendent motivation". Discussion. The respondents from the first group were carried and born in 1972-1973 (the epoch of "stability" and "stagnation" in Russia). The respondents from the second group were carried and born in 1986-1987, just after the beginning of "perestroika" in society. Perestroika gave an impetus to the development of personality including gender sphere. The second group reports a higher level of need for independent and positive activity, and these tendencies are clearer in girls.
\end{abstract}

Key words: perestroika, personality psychology, stress, gender differences, self-regulation, self-realization.

\section{Introduction}

The development of society undergoes the periods of transformations from time to time. For people, it means a necessity to change abruptly the previous standards of behaviour as a response to new conditions of changed socio-economic reality.

This period in Russia dated by the end of the XX century was called "perestroika" (rearrangement). It began in 1985, when the leaders of the country suggested replacing ideological dominance of one party to wide democratic procedures.

In our research, the notion "gender" is used in a broad sense. We agree that "gender" is defined by culture, while "sex differences" are, as a whole, genetically determined. We cannot assume that in the transitive society, sex differences change substantially. "Gender 
stereotypes", in turn, may see some important changes (especially in our case, which is associated with the phenomenon of "perestroika" in Russia). We assume that in the self-report type instruments (including the 16-PF), the changed social reality will have a substantial influence over the choice of an answer.

\section{The goal of the study}

The goal of the study is to investigate the influence of the processes of social transformation of society on the change of gender stereotypes of youngsters.

\section{The design of the study}

This publication is a part of a wide-scale study of 1992-2018 (the analysis of more than 5,000 psycho-diagnostic records and social questionnaires) devoted to the influence of social changes in the Russian society on the formation of youngsters' personality. The data are being generalized and published now; there have been no reports of the results of intermediate studies earlier.

The article juxtaposes the results of two psycho-diagnostic techniques applied on two groups of respondents: R.B. Cattell's 16-PF Questionnaire and the Motivation Induction Method (MMI) suggested by Joseph Nuttin.

The first group of respondents (the second year medical students of the department of general medicine, Yaroslavl State Medical University) has been examined in 1992 (the publication contains the data of 120 persons in total - 60 boys and 60 girls). They were 19 years old during psycho-diagnostics, i.e. the first group is a group of respondents born in 1973 (the "pre-perestroika generation"). Their pre-natal and post-natal development took place in the time of sustainable development of the country, and, importantly, in the time when the state provided a large social support to individuals including the aid in bringing children up. On the one hand, this provided social-psychological comfort; on the other hand, the state unintentionally created parasitical attitudes and infantile responses to social life. The main challenge was that the process of development of the state was hampered by individuals' low activity. All the problems were transferred to some abstract "official" (up to maintaining order in one's own courtyard). Our respondents (born in 1973) reached the age of 12 before the "perestroika" era, which, in the opinion of a whole range of scientists, largely shaped their personality (through learning both social values and the values of family microenvironment) [1-3].

Another group of respondents (the second year medical students of the department of general medicine, Yaroslavl State Medical University) has been examined in 2006 (the publication contains the data of 120 persons as well - 60 boys and 60 girls, the groups are levelled by their structure as thoroughly as possible). They were 19 years old during psychodiagnostics (the "perestroika generation"). After their birth, the respondents from the second group (born in 1987) found themselves in a social situation, which some scientists are likely to call "social chaos". Social protection of personality from the state substantially weakened and, simultaneously, social-psychological burden on a particular personality significantly grew and the demands for its initiative and responsibility became higher. Moreover, the experience of the past life of parents was not relevant anymore, since there was quite another reality establishing in society. As we can see, the first 12 years of life in the "perestroika generation" were completely different. In the study, we tried to figure out how it influenced the formation of a personality and its gender peculiarities and what was the direction of these changes [4-6]. 
Table 1. The dynamics of the values of the 16-PF factors (raw scores) in the first block of personal characteristics (Mann-Whitney U-test).

\begin{tabular}{|c|r|c|c|c|c|c|}
\hline \multirow{2}{*}{ 16-PF factors } & \multicolumn{3}{|c|}{ Boys } & \multicolumn{3}{c|}{ Girls } \\
\cline { 2 - 7 } & 1992 & 2006 & $\mathrm{p}$ & 1992 & 2006 & $\mathrm{p}$ \\
\hline $\mathrm{A}$ & 9.53 & 11.80 & 0.01 & 11.18 & 13.48 & 0.001 \\
\hline $\mathrm{F}$ & 15.85 & 14.70 & - & 15.88 & 15.57 & - \\
\hline $\mathrm{H}$ & 12.67 & 14.38 & 0.05 & 13.20 & 14.47 & - \\
\hline
\end{tabular}

Table 2. The dynamics of the values of the 16-PF factors (raw scores) in the second block of personal characteristics (Mann-Whitney U-test).

\begin{tabular}{|c|c|r|c|r|r|c|}
\hline \multirow{2}{*}{ 16-PF factors } & \multicolumn{3}{|c|}{ Boys } & \multicolumn{3}{c|}{ Girls } \\
\cline { 2 - 8 } & 1992 & 2006 & $\mathrm{p}$ & 1992 & 2006 & $\mathrm{p}$ \\
\hline $\mathrm{E}$ & 13.88 & 13.82 & - & 11.82 & 13.67 & 0.01 \\
\hline $\mathrm{G}$ & 11.43 & 11.13 & - & 12.37 & 12.37 & - \\
\hline $\mathrm{L}$ & 9.75 & 11.43 & 0.01 & 9.03 & 11.55 & 0.001 \\
\hline N & 8.37 & 8.83 & - & 9.83 & 9.57 & - \\
\hline Q2 & 9.07 & 9.70 & - & 7.20 & 8.80 & 0.05 \\
\hline
\end{tabular}

Table 3. The dynamics of the values of the 16-PF factors (raw scores) in the third block of personal characteristics (Mann-Whitney U-test).

\begin{tabular}{|c|r|r|c|r|r|c|}
\hline \multirow{2}{*}{ 16-PF factors } & \multicolumn{3}{|c|}{ Boys } & \multicolumn{3}{c|}{ Girls } \\
\cline { 2 - 7 } & 1992 & 2006 & $\mathrm{p}$ & 1992 & 2006 & $\mathrm{p}$ \\
\hline $\mathrm{B}$ & 7.83 & 8.55 & 0.05 & 7.82 & 8.10 & - \\
\hline $\mathrm{E}$ & 13.88 & 13.82 & - & 11.82 & 13.67 & 0.01 \\
\hline $\mathrm{M}$ & 10.67 & 10.03 & - & 11.25 & 9.47 & 0.01 \\
\hline $\mathrm{N}$ & 8.37 & 8.83 & - & 9.83 & 9.57 & - \\
\hline $\mathrm{Q} 1$ & 9.07 & 10.70 & 0.001 & 8.00 & 9.38 & 0.01 \\
\hline
\end{tabular}

\section{Results and discussion}

The experience of the application of R.B. Cattell's questionnaire enables us to unite the groups of traits in five main blocks and rely on them in comparing the personality's response to a drastic change of circumstances.

The first block of personality's traits (Table 1) involves the characteristics regulating personality's behaviour in a wide social context: A+ ("sociability", "communicative competence"), F+ ("impulsivity", "high optimism"), H+ ("courage", "freedom to enter contacts"). We see boys' increased activity immediately in the sphere of communicative competences and the sphere of wide social contacts. Girls report significant increase only in the sphere of communicative competences; however, the increase more than substantial by 3.2 raw scores.

The second block (Table 2) reflects the relations in micro-society: E+ ("activity, initiative"), G+ ("responsibility in relations"), L- ("vulnerability", "suspiciousness", "envy"), N+ ("cautiousness", "diplomacy, "prudence"), Q2+ ("independence from a group", "quick wit", "generating one's solutions"). Boys report statistically significant increase in the factor associated with the growth of mental tension. Girls report statistically significant increase in the same factor (though in a greater degree) as well as in the indices of general activity growth and the growth of independence of deeds from the group opinion.

The third block (Table 3) contains a set of personal qualities providing intellectual success (in a broad sense): B+ ("ability to catch the information quickly", "abstractedness, logic"), E+ ("activity, initiative"), M+ ("imagination"), N+ ("cautiousness", "diplomacy", 
Table 4. The dynamics of the values of the 16-PF factors (raw scores) in the fourth block of personal characteristics (Mann-Whitney U-test).

\begin{tabular}{|c|r|r|c|c|c|c|}
\hline \multirow{2}{*}{ 16-PF factors } & \multicolumn{3}{|c|}{ Boys } & \multicolumn{3}{c|}{ Girls } \\
\cline { 2 - 7 } & 1992 & 2006 & $\mathrm{p}$ & 1992 & 2006 & $\mathrm{p}$ \\
\hline $\mathrm{C}$ & 13.62 & 15.05 & - & 14.08 & 15.37 & - \\
\hline $\mathrm{I}$ & 9.38 & 9.17 & - & 14.85 & 12.67 & - \\
\hline O & 11.63 & 10.07 & 0.05 & 13.38 & 12.00 & 0.05 \\
\hline Q4 & 11.55 & 12.30 & - & 13.72 & 12.97 & - \\
\hline
\end{tabular}

Table 5. The dynamics of the values of the 16-PF factors (raw scores) in the fifth block of personal characteristics (Mann-Whitney U-test).

\begin{tabular}{|c|c|c|c|c|c|c|}
\hline \multirow{2}{*}{ 16-PF factors } & \multicolumn{3}{|c|}{ Boys } & \multicolumn{3}{c|}{ Girls } \\
\cline { 2 - 7 } & 1992 & 2006 & $\mathrm{p}$ & 1992 & 2006 & $\mathrm{p}$ \\
\hline E & 13.88 & 13.82 & - & 11.82 & 13.67 & 0.01 \\
\hline G & 11.43 & 11.13 & - & 12.37 & 12.37 & - \\
\hline H & 12.67 & 14.38 & 0.05 & 13.20 & 14.47 & - \\
\hline Q3 & 10.13 & 11.78 & 0.01 & 10.68 & 11.32 & - \\
\hline
\end{tabular}

“prudence"), Q1 (independence, radical judgements). The table shows the increased burden in using formal intelligence and independence of judgements in boys. Girls report the increased values of as much as three out of five factors related to this group: the general activity of thinking processes, imagination and, like boys, the independence of judgements from the opinion of others.

The fourth block (Table 4) contains personal factors "closely associated with the emotional regulation of behaviour": C+ ("power of I"), I+ ("emotional sensibility, vulnerability"), $\mathrm{O}+$ ("proneness to a sense of guilty, restlessness, sensibility to external approval"), Q4 ("high tension", "frustration", excessive fatigue, irritability). From the standpoint of gender, we reveal no differences. Both boys and girls report the increased factor O - "experiences of guilt", "reduced feeling of safety", "vulnerability".

Finally, we are interested in the dynamics of the fifth block scales (Table 5) - the group of personal traits, which directly provide voluntary regulation of behavior: E+ ("activity, initiative"), G+ ("strong super Ego"), H+ ("social boldness"), Q3+ ("control of wishes and awareness of social demands to deeds", and "a gyroscopic factor of a personality" as figuratively described by R.B. Cattell). This is the only "block" among the "blocks" under examination, which does not have any single tendencies for both genders. Boys report the increased indices of social boldness and self-control, while girls show the increased indices of general activity and initiative.

The data of the Joseph Nuttin's inventory can be a meaningful addition to the tendencies under consideration (a projective technique of the "uncompleted sentences" type aimed at studying the motives of personality's behavior) [7,8]. The results are evaluated by two parameters: "values" and "time" perspective (in its turn, it is subdivided into "real" time measured by calendars/devices and "social" time closely associated with its experiencing by a subject of life and activity - childhood/adulthood/old age...).

Boys and girls report unidirectional increase in the indices of the values of self-realization and productive activity values and reduction of the value of communication (relations in society) itself in the system of basic classes (Table 6 ) of values ( $\mathrm{S}$ - values of the personality itself; SR - the issues of self-realization; $\mathrm{R}$ - work, productive activity; $\mathrm{C}$ - communication; $\mathrm{E}$ - cognitive and research motivation; $\mathrm{T}$ - transcendent motivation; $\mathrm{P}$ - possession of something; L - recreation, leisure, pleasures). 
Table 6. The dynamics of the values of the MMI inventory factors - value orientations (Mann-Whitney U-test).

\begin{tabular}{|c|c|c|c|r|c|c|}
\hline \multirow{2}{*}{ MMI factors } & \multicolumn{3}{|c|}{ Boys } & \multicolumn{3}{c|}{ Girls } \\
\cline { 2 - 7 } & 1992 & 2006 & $\mathrm{p}$ & 1992 & 2006 & $\mathrm{p}$ \\
\hline S. & 9.95 & 9.10 & - & 10.38 & 8.85 & 0.05 \\
\hline SR & 6.05 & 8.65 & 0.001 & 5.55 & 9.20 & 0.001 \\
\hline R. & 4.65 & 6.03 & 0.01 & 4.22 & 5.53 & 0.01 \\
\hline C & 8.30 & 6.30 & 0.01 & 10.38 & 8.37 & 0.01 \\
\hline E & 1.12 & 0.60 & 0.05 & 0.80 & 0.90 & - \\
\hline T & 1.52 & 0.70 & 0.001 & 0.90 & 0.73 & - \\
\hline P & 2.17 & 1.38 & - & 0.98 & 0.67 & - \\
\hline L & 3.13 & 3.07 & - & 4.23 & 2.62 & 0.001 \\
\hline
\end{tabular}

Table 7. The dynamics of the values of the MMI inventory factors - "real time" (Mann-Whitney U-test).

\begin{tabular}{|c|r|r|c|r|r|c|}
\hline \multirow{2}{*}{ MMI factors } & \multicolumn{3}{|c|}{ Boys } & \multicolumn{3}{c|}{ Girls } \\
\cline { 2 - 7 } & 1992 & 2006 & $\mathrm{p}$ & 1992 & 2006 & $\mathrm{p}$ \\
\hline $\mathrm{T}$ & 1.10 & 1.47 & 0.01 & 0.50 & 0.53 & - \\
\hline $\mathrm{D}$ & 0.58 & 0.60 & - & 0.30 & 0.50 & - \\
\hline $\mathrm{W}$ & 0.15 & 0.22 & - & 0.42 & 0.13 & 0.01 \\
\hline $\mathrm{M}$ & 1.38 & 1.28 & - & 1.97 & 1.13 & 0.01 \\
\hline $\mathrm{Y}$ & 12.78 & 12.18 & - & 12.65 & 13.45 & 0.05 \\
\hline
\end{tabular}

Table 8. The dynamics of the values of the MMI inventory factors - "social time" (Mann-Whitney U-test).

\begin{tabular}{|c|c|c|c|c|c|c|}
\hline \multirow{2}{*}{ MMI factors } & \multicolumn{3}{|c|}{ Boys } & \multicolumn{3}{c|}{ Girls } \\
\cline { 2 - 8 } & 1992 & 2006 & $\mathrm{p}$ & 1992 & 2006 & $\mathrm{p}$ \\
\hline $\mathrm{P}$ & 0.22 & 0.52 & 0.01 & 0.48 & 0.40 & - \\
\hline $\mathrm{E}$ & 4.28 & 5.63 & 0.01 & 5.53 & 5.12 & - \\
\hline $\mathrm{A}$ & 2.37 & 0.35 & 0.001 & 0.52 & 0.27 & 0.05 \\
\hline $\mathrm{O}$ & 0.23 & 0.25 & - & 0.12 & 0.17 & - \\
\hline $\mathrm{L}$ & 0.70 & 1.27 & 0.001 & 1.47 & 2.40 & 0.001 \\
\hline $\mathrm{I}$ & 7.90 & 7.73 & - & 7.87 & 7.65 & - \\
\hline
\end{tabular}

Boys report a significantly decreased value of cognitive and research activity and transcendent motivation, while girls demonstrate a statistically significant drop of interest in self-analysis and the values of recreation.

From the standpoint of real time (Table 7), boys began to prefer activity "in real time" (T), while girls - in quite remote time: "year and more" (Y), the interest to the "week" (W) and "month" (M) scale has reduced.

The wishes in the day (D) scale have not changed.

In "social time" (Table 8), the changes are more substantial: a general increased tendency to see values in the scale of a whole life (L) and decreased value of the "adulthood" alone (A).

Boys report the increased value of rethinking "the past" $(\mathrm{P})$ and activity "in the present" (E, time of study).

The values of the periods of "old age" $(\mathrm{O})$ and "the open present" (I, the expressions like "to be beautiful", i.e. now and always) have not changed. 


\section{Conclusions}

1. The study has registered a substantial change of personal characteristics in the "perestroika generation" compared to the "pre-perestroika generation" (within a framework of the groups under study). The changes of the following characteristics can be called substantial for a new generation: "increased communicability", "increased freedom of judgements from the group influence", and, at the same time, "increased tension in relations with others", "increased sense of safety", increased indices of the "self-realization", "work and productive activity", "decreased values of relations themselves" values, in time perspective - increased values of tasks achieved in a life scale and decreased values of the period of adulthood alone.

2. Against this background, we register gender differences, which are most clearly manifested in girls. Apart from these general changes, we mark a significant increase of general life activity (exceeding the analogous index in boys) and characteristics of applying "intelligence in general", increased independence of deeds from the group opinion, increased participation of imagination in planning activity, decreased interest in self-analysis and values of recreation, and increased interest in planning their activity for year and more. We should note that girls have more changes in "real time", while boys in "social time".

We can interpret the sum of described changes as a decisive step to the substantial correction of a traditional passive gender stereotype of girl's behaviour and realization of new significant opportunities given by the perestroika situation.

3. In boys, we rather observe the sharpening and development of a traditional stereotype of behaviour; however, we can predict the effect of this correction as positive, it is directed at the increasing productivity of social activity.

The research was supported by the Russian Humanitarian Science Foundation (RHSF), project 16-06-00960.

\section{References}

[1] N. Howe, W. Strauss, Generations: The History of America's Future, 1584 to 2069 (William Morrow \& Company, New York, 1991)

[2] F. Aboud, A.Yousafzai, Annu. Rev. Psychol. 66, 433-457 (2015)

[3] E. Nikolaeva, Med. psihol. Ross. 2(37), 4 (2016)

[4] N. Klyueva, Med. psihol. Ross. 6(41), 2 (2016)

[5] N. Zhuravleva, Dynamics of Personality's Value Orientations in the Russian Society (Institut Psikhologii RAN, Moscow, 2006)

[6] V. Uryvaev, Med. psihol. Ross. 5(46), 6 (2017)

[7] Zh. Nyutten, Motivation, Action and Perspective of the Future (Smysl, Moscow, 2004)

[8] N. Tolstykh, Chronotop: culture and ontogenesis (Universum, Moscow, 2010) 